\title{
alteração das Características Biológicas dos Biótipos de AZEVÉm (Lolium multiflorum) OCASIONADA PELA RESISTÊNCIA AO HERBICIDA GLYPHOSATE ${ }^{1}$
}

\author{
Change in the Biological Characteristics of Ryegrass (Lolium multiflorum) Biotypes Caused by \\ Resistance to the Herbicide Glyphosate
}

VARGAS, L. ${ }^{2}$, ROMAN, E.S. ${ }^{3}$, RIZZARDI, M.A. ${ }^{4}$ e SILVA, V.C. ${ }^{5}$

\begin{abstract}
RESUMO - A resistência de biótipos de azevém ao herbicida glyphosate está alterando o manejo da vegetação de cobertura do solo em pomares de maçã. O objetivo deste trabalho foi determinar a dose de glyphosate necessária para reduzir $50 \%$ do acúmulo de matéria seca $\left(\mathrm{GR}_{50}\right)$, a resposta do biótipo resistente e sensivel a herbicidas graminicidas e o acúmulo de matéria seca destes biótipos durante o ciclo. Para isso, foram conduzidos três experimentos. No primeiro, os tratamentos constaram de doses crescentes de glyphosate aplicadas sobre plantas dos biótipos resistente e sensivel para determinar o $\mathrm{GR}_{50}$. No segundo experimento, os tratamentos constaram de doses dos herbicidas glyphosate, haloxyfop-r, diclofop, fluazifop-p, fenoxaprop-p e paraquat. No terceiro experimento, sementes dos biótipos resistente e sensivel foram semeadas em recipientes com capacidade para $10 \mathrm{~L}$ e as plantas originadas delas foram colhidas quinzenalmente, para determinação da matéria seca da parte aérea, radicular e total. Como resultados, foi obtido $\mathrm{GR}_{50}$ de 287,5 e de 4.833,5 g e.a. ha ${ }^{-1}$ de glyphosate para os biótipos sensivel e resistente, respectivamente, e verificou-se que existem diferenças significativas na resposta dos biótipos aos herbicidas graminicidas, dependendo da dose utilizada. Além disso, o biótipo sensivel evidenciou maior capacidade de acúmulo de matéria seca e produção de sementes. Constatou-se, assim, fator de resistência (FR) de 16,8 e que o mecanismo de resistência provoca alterações nas características biológicas do biótipo resistente e afeta a sensibilidade deste aos herbicidas graminicidas.
\end{abstract}

Palavras-chave: inibidores da EPSPs, graminicidas, manejo, resistência, maçã.

\begin{abstract}
The identification of ryegrass biotypes resistant to glyphosate is causing changes in weed management in apple orchards. Three experiments were carried out to determine the $G R_{50}$ of the biotypes, to grass herbicides and dry matter accumulation. The first experiment tested different rates of glyphosate. Their effects on $G R_{50}$ dry matter accumulation by the biotype were assessed. In the second experiment different rates of glyphosate and grass herbicides were tested: glyphosate, haloxyfop-r, diclofop, fluazifop-p, fenoxaprop-p and paraquat. A third experiments was carried out under glasshouse conditions to determine the curve of dry matter accumulation. The results showed $G R_{50}$ of 287.5 and $4,833.5 \mathrm{~g} \mathrm{e.} \mathrm{a.} \mathrm{ha-1} \mathrm{of} \mathrm{glyphosate} \mathrm{for} \mathrm{the}$ sensitive and resistant biotypes, respectively. The results showed that the resistant factor (RF) was 16.8 and that the resistance mechanism alters the biological characteristics of the resistant biotype affecting its sensitivity to grass herbicides.
\end{abstract}

Key words: herbicides, EPSP inhibitors, grass herbicides, apple orchards.

Recebido para publicação em 13.10.2004 e na forma revisada em 21.4.2005.

2 Eng.-Agr., D.S., Pesquisador da Embrapa Trigo, Caixa Postal 451, 99001-970 Passo Fundo-RS, <leandro@cnpuv.embrapa.br>;

${ }^{3}$ Eng.-Agr., Ph.D., Pesquisador da Embrapa Trigo, <eroman@enpt.embrapa.br>. ${ }^{4}$ Eng.-Agr., D.S., Professor da Faculdade de Agronomia e Medicina Veterinária da Universidade de Passo Fundo - UPF, 99001-970 Passo Fundo-RS, <rizzardi@upf.tche.br>. ${ }^{5}$ Biólogo, Laboratorista da Embrapa Uva e Vinho, Caixa Postal 1513, 95200-000 Vacaria-RS. 


\section{INTRODUÇÃO}

O glyphosate é o principal herbicida utilizado para controle da vegetação de cobertura do solo em pomares de maçã. $\mathrm{Na}$ região de Vacaria-RS, ele é utilizado há mais de 15 anos e, de forma geral, são realizadas entre três e quatro aplicações do produto por ano; a dose utilizada normalmente situa-se entre 720 e 1.080 g e.a. ha ${ }^{-1}$.

O uso repetido de glyphosate deve-se, principalmente, ao fato de que esse produto apresenta alta eficiência e custo relativamente baixo. $\mathrm{O}$ uso indiscriminado de herbicidas propiciou o desenvolvimento de muitos casos de resistência a tais compostos por diversas espécies daninhas (Burnside, 1992). A resistência é a capacidade adquirida de uma planta de sobreviver a determinados tratamentos herbicidas que, sob condições normais, controlam os integrantes da população (Weed, 1999).

O número de espécies de plantas daninhas resistentes ao glyphosate está aumentando; atualmente, são reconhecidas seis espécies resistentes em 20 diferentes regiões (Weed, 2004). O primeiro caso de azevém (Lolium multiflorum) resistente ao glyphosate foi relatado por Perez \& Kogan (2002). O biótipo resistente foi identificado em pomares, no Chile, que vinham recebendo, em média, três aplicações de glyphosate por ciclo, durante os últimos 10 anos (Perez \& Kogan, 2002). Segundo Powles et al. (1998), após 15 anos de uso bem sucedido de glyphosate na Austrália, foram identificados biótipos de Lolium rigidum resistentes a esse herbicida.

No Brasil, foram identificados biótipos de azevém (Lolium multiflorum) resistentes ao glyphosate em lavouras de culturas anuais e em pomares (Roman et al., 2004). Em todos esses casos a aplicação repetida e continuada de glyphosate para controle da vegetação é considerada a principal causa da seleção dos biótipos resistentes.

O mecanismo que confere resistência ao glyphosate em Lolium rigidum ainda não foi determinado com clareza. As pesquisas realizadas por Lorraine-Colwill et al. (2003) encontraram diferenças na translocação do glyphosate entre os biótipos resistentes e sensiveis.

Planta Daninha, Viçosa-MG, v. 23, n. 1, p. 153-160, 2005
O controle de azevém resistente ao glyphosate está sendo realizado principalmente com uso de herbicidas graminicidas, dependendo da cultura implantada. Entretanto, é possivel observar, a campo, que existem diferenças na evolução dos sintomas e no período para o controle dos biótipos resistentes e sensiveis entre os diferentes graminicidas, sugerindo que o mecanismo que confere resistência ao glyphosate possa estar interferindo na atividade destes herbicidas. A absorção e a translocação do glyphosate e dos herbicidas haloxyfop, sethoxydim e fluazifop-p foram avaliadas por Harker \& Dekker (1988). Estes autores avaliaram o controle de Agropyron repens em diferentes estádios vegetativos, e os resultados evidenciaram que todos os herbicidas translocaram-se igualmente, porém o haloxyfop distribuiu-se de forma mais uniforme nos rizomas, o que proporcionou maior eficiência a este herbicida.

O azevém é uma espécie anual de inverno que se caracteriza como a planta daninha mais comum em pomares de maçã. A ocorrência de biótipos de azevém resistentes ao glyphosate está dificultando o controle desta espécie, uma vez que o número de produtos registrados para a cultura da maçã - com potencial de uso neste caso - é pequeno. Assim, os produtores obrigam-se a alterar o manejo da vegetação nessas áreas, utilizando métodos de manejo e controle menos eficientes e com maior custo de aplicação. Até o momento, não há informações sobre o mecanismo que confere a resistência, bem como sobre os possiveis efeitos deste mecanismo na atividade de outros herbicidas e no comportamento biológico do biótipo resistente. Assim, o objetivo deste trabalho foi determinar a dose de glyphosate necessária para reduzir $50 \%$ do acúmulo de matéria seca $\left(\mathrm{GR}_{50}\right)$, a resposta dos biótipos resistente e sensivel a herbicidas graminicidas e o acúmulo de matéria seca desses biótipos durante o ciclo.

\section{MATERIAL E MÉTODOS}

Foram conduzidos três experimentos, em casa de vegetação, na Estação Experimental da Embrapa Uva e Vinho, em Vacaria-RS, durante os anos de 2003 e 2004.

Os tratamentos herbicidas foram aplicados com aspersor costal de precisão, com 
volume de calda de $150 \mathrm{~L} \mathrm{ha}^{-1}$. O delineamento experimental adotado foi o completamente casualizado, com quatro repetições. A toxicidade dos tratamentos herbicidas foi avaliada utilizando-se escala percentual, em que a nota 0 significou nenhum efeito de dano às plantas e a nota 100 representou morte ou completa supressão destas.

As sementes utilizadas nos experimentos foram provenientes de um pomar de maçã localizado em Vacaria-RS. Em todos os experimentos foram semeadas dez sementes de azevém em cada recipiente com capacidade para $500 \mathrm{~mL}$; após a emergência das plantas, procedeu-se ao desbaste, deixando-se quatro plantas por vaso/repetição.

No primeiro experimento, realizado para determinar o $\mathrm{GR}_{50}$, foram aplicadas doses crescentes do herbicida glyphosate $(0,45,90,180$, $270,360,540,720,1.080,1.440,2.160,2.880$, $3.600,4.320,5.040,5.760,7.200,8.640$ e 10.080 g e.a. ha ${ }^{-1}$ ), quando a maioria das plantas atingiu o estádio vegetativo de três a quatro folhas. Aos 25 dias após o tratamento (DAT), as plantas foram colhidas e, após a secagem em estufa, a uma temperatura de $60{ }^{\circ} \mathrm{C}$, até atingir peso constante, foi determinada a matéria seca produzida em resposta às referidas doses do herbicida.

No segundo experimento, foram aplicados os herbicidas glyphosate (180 e 360 g e.a. ha-1), haloxyfop-r (12, 25 e $150 \mathrm{~g}^{-1}$ ), diclofop (140, 284 e $\left.426 \mathrm{~g} \mathrm{ha}^{-1}\right)$, fluazifop-p $(45,90$ e $\left.187 \mathrm{~g} \mathrm{ha}^{-1}\right)$, fenoxaprop-p (25, 55 e $\left.110 \mathrm{~g} \mathrm{ha}^{-1}\right)$ e paraquat $\left(200 \mathrm{~g} \mathrm{ha}^{-1}\right)$, quando a maioria das plantas atingiu o estádio de três a quatro folhas.

No terceiro experimento, foram semeadas dez sementes de azevém em recipientes com capacidade para 10 L. Após a emergência das plantas, procedeu-se ao desbaste, deixando-se duas plantas por vaso. Após a emergência das plantas, a cada 15 dias, foram colhidas oito plantas (quatro vasos / repetições) e, após secagem em estufa, a uma temperatura de $60^{\circ} \mathrm{C}$, até atingir peso constante, foi determinada a matéria seca da parte aérea, radicular e total. Avaliou-se ainda, neste experimento, o número de perfilhos produzidos por planta, o número de dias necessários para floração, o número de sementes produzidas por inflorescência, o número total de sementes produzidas por planta e o número de dias necessários para a planta completar o ciclo. Em todas essas avaliações foram utilizados quatro vasos / repetições contendo duas plantas.

Os dados obtidos nos experimentos foram submetidos à análise de variância e, quando constatada significância, procedeu-se à complementação da análise. Para comparação de médias, utilizou-se o teste de Duncan a 5\% de probabilidade. Já para cálculo do $\mathrm{GR}_{50}$ utilizou-se o software TableCurve $2 \mathrm{D}$, ajustandose ao modelo logístico dose/resposta.

\section{RESULTADOS E DISCUSSÃO}

As curvas de dose-resposta, construídas com os valores de matéria seca produzida pelos biótipos, em resposta às doses crescentes de glyphosate, resultaram em $\mathrm{GR}_{50}$ de 287,5 g e.a. ha ${ }^{-1}$ de glyphosate para o biótipo sensivel e de $4.833,5$ g e.a. ha- ${ }^{-1}$ para o resistente (Figuras 1 e 2). Esses valores indicam um Fator de Resistência (FR), relação entre o $\mathrm{GR}_{50}$ do biótipo resistente e o do sensível, de 16,8 , ou seja, indica que a dose do herbicida glyphosate necessária para reduzir 50\% da produção de matéria seca do biótipo resistente é 16,8 vezes maior do que aquela necessária para produzir o mesmo efeito sobre o biótipo sensivel. Pratley et al. (1999) e Lorraine-Colwill et al. (2003) identificaram biótipos de Lolium rigidum dez vezes mais resistentes ao glyphosate. Roman et al. (2004) obtiveram controle do biótipo sensível de azevém (Lolium multiflorum) com a dose de 360 g e.a. ha ${ }^{-1}$ de glyphosate, enquanto a dose de 5.760 g e.a. ha-1 não controlou o biótipo resistente, produzindo toxicidade de $45 \%$. As diferenças encontradas entre os autores podem estar relacionadas com as diferentes condições ambientais, diferentes formulações do glyphosate e demais condições de aplicação e crescimento dos biótipos.

Roman et al. (2004) relatam que os herbicidas graminicidas inibidores da enzima acetil-CoA carboxilase (ACCase), haloxyfop-r e diclofop, controlam igualmente o biótipo sensível e o resistente quando aplicados no estádio de três a quatro folhas. Contudo, em geral, observa-se que o tempo necessário para ocorrer a morte do biótipo resistente, em resposta a 
herbicidas graminicidas, é maior do que aquele requerido para o biótipo sensivel. Assim, para avaliar essa diferença, foi conduzido o segundo experimento, em casa de vegetação, utilizando-se diferentes doses de herbicidas.

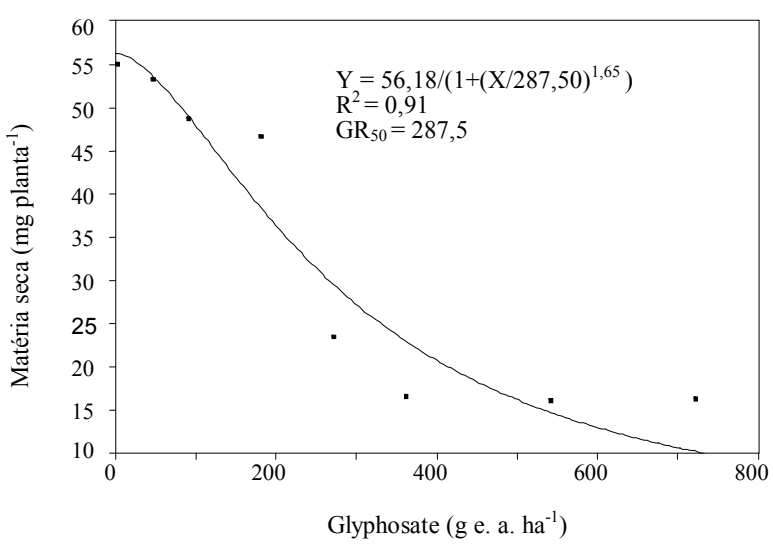

Figura 1 - Produção de matéria seca e $\mathrm{GR}_{50}$ do biótipo sensível de azevém em resposta a doses crescentes de glyphosate. Embrapa Uva e Vinho, Vacaria-RS, 2004.

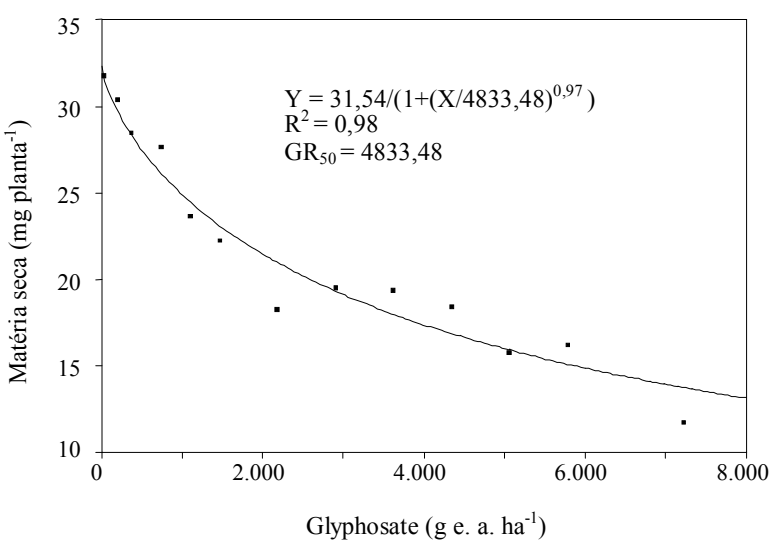

Figura 2 - Produção de matéria seca e $\mathrm{GR}_{50}$ do biótipo resistente de azevém em resposta a doses crescentes de glyphosate. Embrapa Uva e Vinho, Vacaria-RS, 2004.

Os resultados evidenciam, aos 45 DAT, que as menores doses dos herbicidas haloxyfop-r, diclofop e fenoxaprop-p controlaram o biótipo sensivel e provocaram toxicidade de 60,75 e $76 \%$, respectivamente, no biótipo resistente (Tabela 1). Já a menor dose do herbicida fluazifop-p evidenciou comportamento inverso, ou seja, provocou maior toxicidade no biótipo resistente $(90 \%)$ do que no biótipo sensivel ao glyphosate (75\%). Além disso, observou-se que o biótipo sensível foi controlado com a dose de $180 \mathrm{~g}$ e.a. ha ${ }^{-1}$ de glyphosate aos 45 DAT, enquanto de 360 g e.a. ha ${ }^{-1}$ não provocou sintomas de toxidez no biótipo resistente. O herbicida paraquat controlou os biótipos sensivel e resistente já aos 10 DAT.

A avaliação do acúmulo de matéria seca, em resposta aos graminicidas (Tabela 2), no geral confirmou os resultados obtidos nas avaliações de toxicidade (Tabela 1). O biótipo sensivel produziu quantidade de matéria seca superior à do resistente no tratamento testemunha (Tabela 2). Entretanto, nos tratamentos com glyphosate, haloxyfop-r (12 $\left.\mathrm{g} \mathrm{ha}^{-1}\right)$, diclofop (140 $\left.\mathrm{g} \mathrm{ha}^{-1}\right)$ e fenoxaprop-p (25 $\left.\mathrm{g} \mathrm{ha}^{-1}\right)$ essa relação inverteu-se, ou seja, nestes tratamentos o biótipo resistente, devido ao efeito do herbicida sobre o biótipo sensivel, produziu maior quantidade de matéria seca. Os tratamentos com as demais doses desses produtos apresentaram produção de matéria seca semelhante (Tabela 2). Contudo, o herbicida fluazifop-p (45 $\mathrm{g} \mathrm{ha}^{-1}$ ), assim como na avaliação de toxicidade, provocou resposta diferenciada dos biótipos, ou seja, neste tratamento o biótipo sensivel produziu maior quantidade de matéria seca (Tabela 2 ).

Esses resultados evidenciam diferenças significativas na resposta dos biótipos aos herbicidas graminicidas e que a magnitude da diferença está em função da dose utilizada. O biótipo resistente ao glyphosate apresentouse, em todas as avaliações, mais tolerante aos herbicidas graminicidas do que o biótipo sensível ao glyphosate, exceção feita ao fluazifop-p. As menores doses dos graminicidas promoveram as maiores diferenças na resposta dos biótipos e o aumento destas igualou a resposta (Tabela 1). O biótipo de Lolium rigidum, resistente ao glyphosate, estudado por Powles et al. (1998), apresentou resistência cruzada ao diclofop e mostrou-se sensivel ao fluazifop e ao sethoxydim.

É importante salientar que no pomar onde foram identificados os biótipos de azevém resistentes ao glyphosate nunca foram utilizados herbicidas graminicidas, descartandose, dessa forma, a possibilidade de estar ocorrendo ou ter ocorrido seleção por parte dos graminicidas. Isso sugere que o mecanismo que confere resistência ao glyphosate é o 
Tabela 1 - Toxicidade aos 10, 25 e 45 dias após tratamentos (DAT) herbicidas em um biótipo de azevém sensível e um resistente ao glyphosate. Embrapa Uva e Vinho, Vacaria-RS, 2004

\begin{tabular}{|c|c|c|c|c|c|c|c|}
\hline \multirow{3}{*}{ Tratamento } & \multirow{3}{*}{$\begin{array}{c}\text { Dose } \\
\left(\mathrm{g} \mathrm{ha}^{-1}\right)\end{array}$} & \multicolumn{6}{|c|}{ Toxicidade (\%) } \\
\hline & & \multicolumn{2}{|c|}{$10 \mathrm{DAT}$} & \multicolumn{2}{|c|}{25 DAT } & \multicolumn{2}{|c|}{$45 \mathrm{DAT}$} \\
\hline & & Sensível & Resistente & Sensível & Resistente & Sensível & Resistente \\
\hline Testemunha & - & $\mathrm{A} 0 \mathrm{~g}$ & $\mathrm{~A} 0 \mathrm{~g}$ & A $0 \mathrm{~g}$ & A $0 \mathrm{f}$ & $\mathrm{A} 0 \mathrm{c}$ & $\mathrm{A} 0 \mathrm{e}$ \\
\hline Paraquat & 200 i.a. & A 100 a & A 100 a & A $100 \mathrm{a}$ & A $100 \mathrm{a}$ & A $100 \mathrm{a}$ & A $100 \mathrm{a}$ \\
\hline Glyphosate & 180 e.a. & A $85 \mathrm{ab}$ & B $0 \mathrm{~g}$ & A $95 \mathrm{ab}$ & $\mathrm{B} 0 \mathrm{f}$ & A $100 \mathrm{a}$ & B $0 \mathrm{e}$ \\
\hline Glyphosate & 360 e.a. & A $92 \mathrm{ab}$ & $\mathrm{B} 0 \mathrm{~g}$ & A $100 \mathrm{a}$ & $\mathrm{BOf}$ & A 100 a & $\mathrm{B} 0 \mathrm{e}$ \\
\hline Haloxyfop-r & 12 i.a. & A $15 \mathrm{fg}$ & A $5 \mathrm{~g}$ & A $45 \mathrm{e}$ & B 10 ef & A 100 a & B $60 \mathrm{~d}$ \\
\hline Haloxyfop-r & 25 i.a. & A $43 \mathrm{~cd}$ & B $18 \mathrm{ef}$ & A $75 \mathrm{~cd}$ & A $65 \mathrm{c}$ & A 100 a & A $100 \mathrm{a}$ \\
\hline Haloxyfop-r & 50 i.a. & A $55 \mathrm{~cd}$ & A $50 \mathrm{c}$ & A $95 \mathrm{ab}$ & A90 ab & A $100 \mathrm{a}$ & A $100 \mathrm{a}$ \\
\hline Diclofop & 140 i.a. & A 25 ef & B $10 \mathrm{fg}$ & $\mathrm{A} 68 \mathrm{~d}$ & B 40d & A $100 \mathrm{a}$ & B 75c \\
\hline Diclofop & 284 i.a. & A $50 \mathrm{~cd}$ & B 30 d & $\mathrm{A} 75 \mathrm{~cd}$ & A $68 \mathrm{c}$ & A $100 \mathrm{a}$ & A $100 \mathrm{a}$ \\
\hline Diclofop & 426 i.a. & A $85 \mathrm{ab}$ & $\mathrm{A} 75 \mathrm{~b}$ & A95ab & A 85b & A 100 a & A $100 \mathrm{a}$ \\
\hline Fluazifop-p & 45 i.a. & A $18 \mathrm{f}$ & A 28 de & B $30 \mathrm{f}$ & A $45 \mathrm{~d}$ & B 75 b & A90 b \\
\hline Fluazifop-p & 90 i.a. & A 40 de & A 45 c & A $80 \mathrm{~cd}$ & A 85b & A 100 a & A 100 a \\
\hline Fluazifop-p & 187 i.a. & A $60 \mathrm{c}$ & A 65 b & A 85 bc & A 85 b & A 100 a & A $100 \mathrm{a}$ \\
\hline Fenoxaprop-p & 25 i.a. & A $25 \mathrm{ef}$ & B $10 \mathrm{fg}$ & A 38 ef & B $20 \mathrm{e}$ & A $100 \mathrm{a}$ & B $76 \mathrm{c}$ \\
\hline Fenoxaprop-p & 55 i.a. & $\mathrm{A} 45 \mathrm{~cd}$ & B 18 ef & $\mathrm{A} 80 \mathrm{~cd}$ & A $68 \mathrm{c}$ & A $100 \mathrm{a}$ & A $100 \mathrm{a}$ \\
\hline Fenoxaprop-p & 110 i.a. & $\mathrm{A} 80 \mathrm{~b}$ & $\mathrm{~A} 70 \mathrm{~b}$ & A $95 \mathrm{ab}$ & A $85 b$ & A $100 \mathrm{a}$ & A $100 \mathrm{a}$ \\
\hline
\end{tabular}

Médias seguidas de letras minúsculas idênticas, na coluna, e médias antecedidas de letras maiúsculas idênticas, na linha, dentro de cada data de avaliação, não diferem significativamente pelo teste de Duncan - $(\mathrm{P}>0,05)$.

i.a.= ingrediente ativo; e.a.= equivalente ácido.

responsável pela alteração da resposta destes biótipos aos inibidores da ACCase avaliados. Além disso, ressalta-se que as doses recomendadas/registradas dos graminicidas avaliados controlam tanto o biótipo de azevém sensivel quanto o biótipo resistente ao glyphosate, descaracterizando a ocorrência de resistência múltipla.

Com base na diferente resposta do biótipo resistente aos herbicidas graminicidas, podese especular que o mecanismo de resistência não está diretamente relacionado com a enzima EPSPs, já que estes herbicidas agem sobre outra enzima, a ACCase, descartando-se as hipóteses de alteração genética na EPSPs e de superprodução de EPSPs. A resistência de Lolium rigidum ao glyphosate não se deve a alterações na EPSPs (Powles et al., 2000). Não há diferença na atividade e na sensibilidade ao glyphosate da enzima EPSPs de biótipos de Lolium rigidum sensivel e resistente (LorraineColwill et al., 2003). Além disso, os biótipos de Lolium rigidum sensivel e resistente ao glyphosate possuem a mesma capacidade para absorver e metabolizar o herbicida.
Tabela 2 - Acúmulo de matéria seca aos 45 dias após tratamentos (DAT) herbicidas em um biótipo de azevém sensível e um resistente ao glyphosate. Embrapa Uva e Vinho, Vacaria-RS, 2004

\begin{tabular}{|c|c|c|c|}
\hline \multirow[t]{2}{*}{ Tratamento } & \multirow{2}{*}{$\begin{array}{l}\text { Dose } \\
\left(\mathrm{g} \mathrm{ha}^{-1}\right)\end{array}$} & \multicolumn{2}{|c|}{$\begin{array}{l}\text { Acúmulo de matéria seca } \\
\qquad\left(\mathrm{mg} \mathrm{planta}^{-1}\right)\end{array}$} \\
\hline & & Sensível & Resistente \\
\hline Testemunha & - & A 368 a & B 315a \\
\hline Paraquat & 200 i.a. & A $54 \mathrm{fg}$ & $\mathrm{A} 40 \mathrm{~h}$ \\
\hline Glyphosate & 180 e.a. & B 60 efg & A 322 a \\
\hline Glyphosate & 360 e.a. & B $51 \mathrm{~g}$ & A 318 a \\
\hline Haloxyfop-r & 12 i.a. & B 72 de & A276b \\
\hline Haloxyfop-r & 25 i.a. & A $63 \mathrm{efg}$ & A $69 \mathrm{fg}$ \\
\hline Haloxyfop-r & 50 i.a. & A $55 \mathrm{fg}$ & A $54 \mathrm{gh}$ \\
\hline Diclofop & 140 i.a. & B 64 efg & A245c \\
\hline Diclofop & 284 i.a. & A 60 efg & A $56 \mathrm{gh}$ \\
\hline Diclofop & 426 i.a. & A $52 \mathrm{~g}$ & A $58 \mathrm{~g}$ \\
\hline Fluazifop-p & 45 i.a. & A $231 \mathrm{~b}$ & B $98 \mathrm{e}$ \\
\hline Fluazifop-p & 90 i.a. & A $81 \mathrm{~d}$ & A $76 \mathrm{f}$ \\
\hline Fluazifop-p & 187 i.a. & A $67 \mathrm{ef}$ & A $70 \mathrm{fg}$ \\
\hline Fenoxaprop-p & 25 i.a. & B 96 c & A225d \\
\hline Fenoxaprop-p & 55 i.a. & A 70 de & A 83 ef \\
\hline Fenoxaprop-p & 110 i.a. & A 62 efg & A $58 \mathrm{~g}$ \\
\hline
\end{tabular}

Médias seguidas de letras minúsculas idênticas, na coluna, e médias antecedidas de letras maiúsculas idênticas, na linha, não diferem significativamente pelo teste de Duncan $(\mathrm{P}>0,05)$.

i.a.= ingrediente ativo; e.a.= equivalente ácido.

Planta Daninha, Viçosa-MG, v. 23, n. 1, p. 153-160, 2005 
Lorraine-Colwill et al. (2003) encontraram evidências de que a resistência de Lolium rigidum ao glyphosate está diretamente relacionada com o aumento da translocação do herbicida para as pontas das folhas. Após a aplicação do glyphosate, os autores verificaram maior translocação do glyphosate para a parte radicular no biótipo sensivel do que no biótipo resistente. Segundo Hartzler (2003), o glyphosate é translocado preferencialmente via xilema no biótipo resistente, acumulando o produto nas folhas mais velhas, de forma semelhante ao que ocorre com os herbicidas triazinas.

Esse comportamento é observado em plantas resistentes após a aplicação do glyphosate. Algumas plantas evidenciam a parte aérea completamente necrosada, porém a região do colo e as raízes permanecem vivas, o que proporciona rebrota, depois de periodos variáveis (Figura 3). Perez \& Kogan (2002) observaram que plantas do biótipo resistente apresentam os meristemas vivos após o tratamento com glyphosate e rebrotam posteriormente. Isso sugere que a translocação do glyphosate até as raízes é limitada no biótipo resistente e que, possivelmente, o transporte via xilema acumula o produto nas extremidades das folhas; assim, os meristemas não são atingidos e a

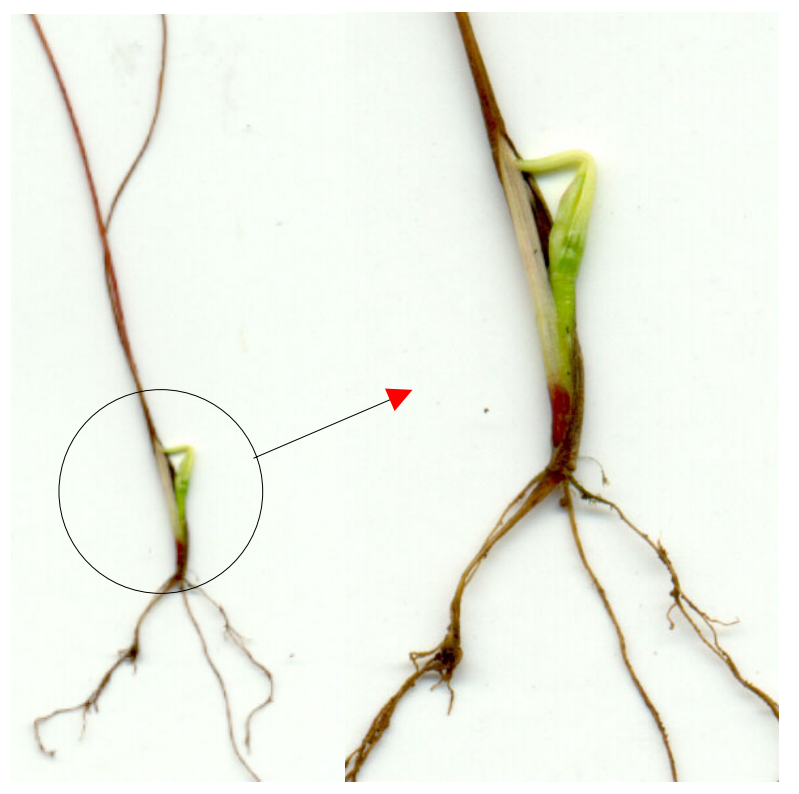

Figura 3 - Detalhe de uma planta de azevém resistente, tratada com 2.880 g e.a. ha ${ }^{-1}$ de glyphosate, rebrotando aos 60 dias após o tratamento com o herbicida. Embrapa Uva e Vinho, Vacaria-RS, 2004. planta rebrota (Figura 3), concordando com Lorraine-Colwill et al. (2003) e Hartzler (2003).

Na Tabela 2, observa-se que o biótipo sensivel acumulou maior quantidade de matéria seca aos 45 DAT. Para testar a hipótese de que o biótipo sensivel acumula maior quantidade de matéria seca do que o resistente, foi realizado experimento em que se avaliou o acúmulo de matéria seca destes biótipos ao longo do ciclo vegetativo.

Na Figura 4 e na Tabela 3 estão apresentados os acúmulos de matéria seca da parte radicular, da parte aérea e total dos biótipos. $\mathrm{O}$ acúmulo de matéria seca da parte radicular foi semelhante entre os biótipos até 75 dias após a emergência (DAE). Na avaliação realizada aos $90 \mathrm{DAE}$, e nas posteriores, o biótipo sensivel apresentou acúmulo de matéria seca nas raizes superior ao do biótipo resistente ao glyphosate. O acúmulo de matéria seca na parte aérea foi semelhante entre os biótipos apenas na primeira avaliação, realizada aos 15 DAE. Em todas as avaliações posteriores, o biótipo sensivel apresentou maior acúmulo de matéria seca na parte aérea. $O$ acúmulo de matéria seca total foi semelhante até 45 DAE. Nas avaliações posteriores, o biótipo sensivel acumulou maior quantidade de matéria seca (Figura 4 e Tabela 3). Powles et al. (2000) observaram que a resistência de Lolium rigidum ao glyphosate é acompanhada por redução da capacidade competitiva.

A menor produção de matéria seca do biótipo resistente na parte aérea está relacionada com o menor número de perfilhos produzidos por este biótipo (Tabela 4). O biótipo sensivel apresentou, em média, 7,2 perfilhos por planta, enquanto o resistente apresentou 4,4 perfilhos. O número de perfilhos interfere diretamente no número de inflorescências da planta, uma vez que cada um produzirá, potencialmente, uma inflorescência. Assim, o número de inflorescências produzidas pelo biótipo sensivel também foi maior do que aquele produzido pelo biótipo resistente. Em conseqüência, o maior número de inflorescências do biótipo sensivel lhe proporcionou maior produção de sementes (Tabela 4). O maior número de sementes produzidas pelo biótipo sensivel deveu-se ao maior número de inflorescências e ao maior número de sementes por inflorescência (Tabela 4). 
Outra característica avaliada foi o número de dias necessários, após a emergência, para que os biótipos iniciassem o período reprodutivo (floração). Observou-se que, em média, o biótipo sensivel floresceu 19 dias antes do biótipo resistente. Já o número de dias necessários para o biótipo sensivel completar o ciclo foi 25 dias menor que o do resistente (Tabela 4).

O maior acúmulo de matéria seca, o maior número de perfilhos, a floração antecipada, a maior produção de sementes e o menor ciclo são características importantes que podem ser utilizadas como ferramentas no manejo e controle da resistência. A adoção de práticas culturais que favoreçam o biótipo sensivel poderá ser uma estratégia eficiente para manejar áreas com resistência.

A capacidade de acumular matéria seca é um importante indicador da capacidade competitiva de uma espécie. Dessa forma, em

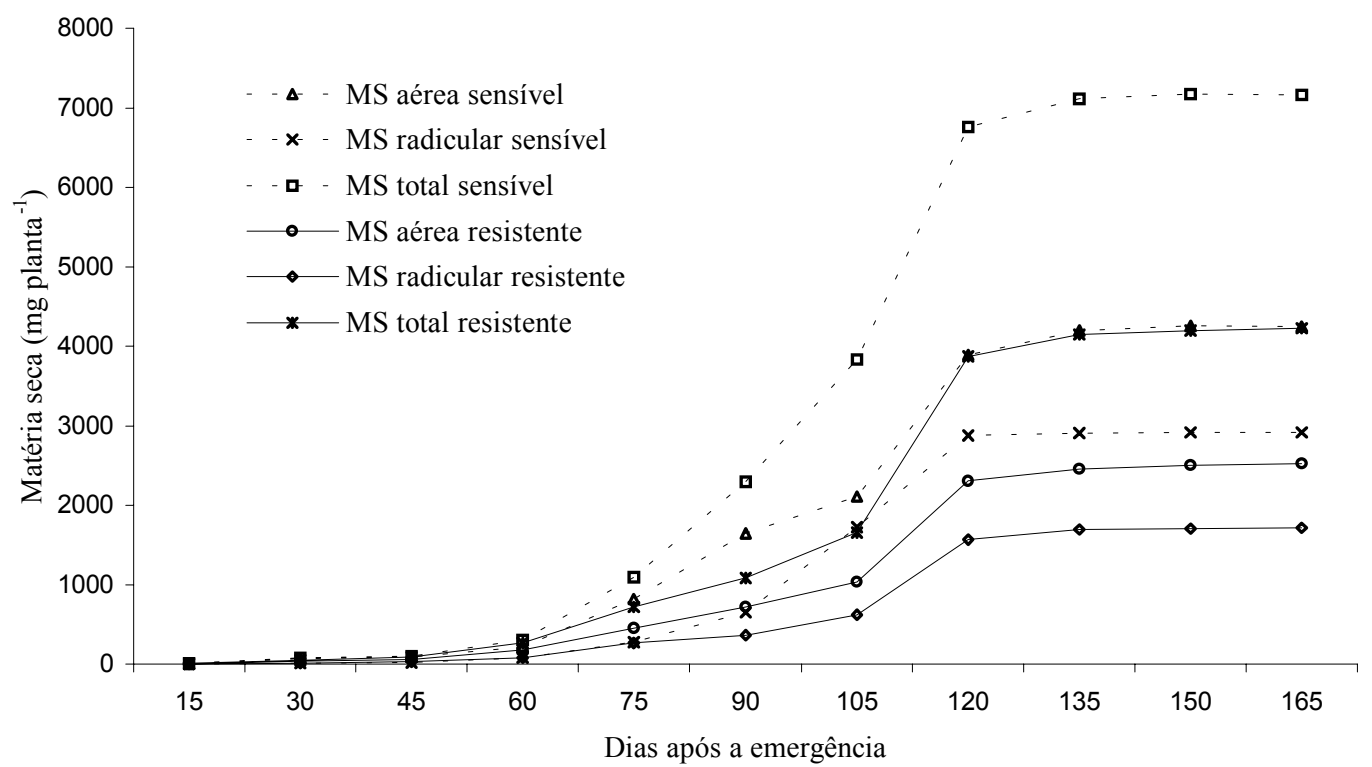

Figura 4 - Curvas de acúmulo de matéria seca total (MS total), parte aérea (MS aérea) e radicular (MS radicular) de um biótipo de azevém sensível e de um resistente ao glyphosate. Embrapa Uva e Vinho, Vacaria-RS, 2004.

Tabela 3 - Produção de matéria seca $\left(\mathrm{mg} \mathrm{planta}^{-1}\right)$ da parte radicular, da parte aérea e total de um biótipo de azevém sensível e um resistente ao glyphosate. Embrapa Uva e Vinho, Vacaria-RS, 2004

\begin{tabular}{|c|c|c|c|c|c|c|}
\hline \multirow{2}{*}{$\mathrm{DAE}^{1 /}$} & \multicolumn{2}{|c|}{ Matéria seca da parte radicular } & \multicolumn{2}{|c|}{ Matéria seca da parte aérea } & \multicolumn{2}{|c|}{ Matéria seca total } \\
\hline & Sensível & Resistente & Sensível & Resistente & Sensível & Resistente \\
\hline 15 & $2,4 \mathrm{a}$ & $3,0 \mathrm{a}$ & $4,3 \mathrm{a}$ & $3,6 \mathrm{a}$ & $6,7 \mathrm{a}$ & $6,6 \mathrm{a}$ \\
\hline 30 & 8,0 a & $12,0 \mathrm{a}$ & $68,0 \mathrm{a}$ & $40,0 \mathrm{~b}$ & $76,0 \mathrm{a}$ & $52,0 \mathrm{a}$ \\
\hline 45 & $15,0 \mathrm{a}$ & $28,0 \mathrm{a}$ & $85,0 \mathrm{a}$ & $59,0 \mathrm{~b}$ & $100,0 \mathrm{a}$ & 87,0 a \\
\hline 60 & $83,0 \mathrm{a}$ & $83,0 \mathrm{a}$ & $221,0 \mathrm{a}$ & $181,0 \mathrm{~b}$ & $304,0 \mathrm{a}$ & $264,0 \mathrm{~b}$ \\
\hline 75 & $280,0 \mathrm{a}$ & $265,0 \mathrm{a}$ & $816,0 \mathrm{a}$ & $450,0 \mathrm{~b}$ & $1.096,0 \mathrm{a}$ & $715,0 \mathrm{~b}$ \\
\hline 90 & $647,0 \mathrm{a}$ & $361,0 \mathrm{~b}$ & $1.648,0 \mathrm{a}$ & $721,0 \mathrm{~b}$ & $2.295,0 \mathrm{a}$ & $1.082,0 \mathrm{~b}$ \\
\hline 105 & $1.724,0 \mathrm{a}$ & $621,0 \mathrm{~b}$ & $2.104,0 \mathrm{a}$ & $1.031,0 \mathrm{~b}$ & $3.828,0 \mathrm{a}$ & $1.652,0 \mathrm{~b}$ \\
\hline 120 & $2.872,0 \mathrm{a}$ & $1.570,0 \mathrm{~b}$ & $3.889,0 \mathrm{a}$ & $2.301,0 \mathrm{~b}$ & $6.761,0 \mathrm{a}$ & $3.871,0 \mathrm{~b}$ \\
\hline 135 & $2.910,0 \mathrm{a}$ & $1.698,0 \mathrm{~b}$ & $4.200,0 \mathrm{a}$ & $2.450,0 \mathrm{~b}$ & $7.110,0 \mathrm{a}$ & $4.148,0 \mathrm{~b}$ \\
\hline 150 & $2.912,0 \mathrm{a}$ & $1.700,0 \mathrm{~b}$ & $4.261,0 \mathrm{a}$ & $2.502,0 \mathrm{~b}$ & $7.173,0 \mathrm{a}$ & $4.202,0 \mathrm{~b}$ \\
\hline 165 & $2.913,0 \mathrm{a}$ & $1.709,0 \mathrm{~b}$ & $4.253,0 \mathrm{a}$ & $2.520,0 \mathrm{~b}$ & $7.166,0 \mathrm{a}$ & $4.229,0 \mathrm{~b}$ \\
\hline
\end{tabular}

${ }^{1 /} \mathrm{DAE}=$ dias após a emergência.

Médias seguidas de letras idênticas, na linha, na avaliação da matéria seca da parte radicular, da matéria seca da parte aérea e da matéria seca total não diferem significativamente pelo teste de Duncan $(\mathrm{P}>0,05)$. 
Tabela 4 - Características biológicas de um biótipo de azevém sensível e um resistente ao glyphosate. Embrapa Uva e Vinho, Vacaria-RS, 2004

\begin{tabular}{|l|r|r|}
\hline \multicolumn{1}{|c|}{ Característica biológica } & $\begin{array}{c}\text { Biótipo } \\
\text { sensível }\end{array}$ & $\begin{array}{c}\text { Biótipo } \\
\text { resistente }\end{array}$ \\
\hline Produção de matéria seca $\left(\mathrm{mg}^{\prime}\right.$ planta $\left.^{-1}\right)$ & $7,1 \mathrm{a}$ & $4,2 \mathrm{~b}$ \\
\hline Número de perfilhos & $7,2 \mathrm{a}$ & $4,4 \mathrm{~b}$ \\
\hline Número de sementes produzidas & $1.382,0 \mathrm{a}$ & $633,0 \mathrm{~b}$ \\
\hline Número de sementes por inflorescência & $192,0 \mathrm{a}$ & $144,0 \mathrm{~b}$ \\
\hline Dias até a floração & $121,0 \mathrm{~b}$ & $140,0 \mathrm{a}$ \\
\hline Ciclo (dias) & $173,0 \mathrm{~b}$ & $198,0 \mathrm{a}$ \\
\hline
\end{tabular}

Médias seguidas de letras idênticas, na linha, não diferem significativamente pelo teste de Duncan $(\mathrm{P}>0,05)$.

condições de competição, em campo, o biótipo sensivel, em tese, possui condições de exercer efeito supressor sobre o crescimento do biótipo resistente. Os biótipos de Senecio vulgaris resistentes às triazinas são menos competitivos que os biótipos sensiveis, em função do dispêndio energético no processo da resistência; assim, os biótipos sensiveis, mais competitivos, tendem a dominar o ambiente (Mortimer, 1998).

A floração antecipada do biótipo sensivel proporciona dessincronia com a floração do biótipo resistente. Apesar de esta característica não impedir totalmente a ocorrência de cruzamentos entre eles, devido ao longo período de floração do azevém e à emissão continuada das inflorescências, ela pode reduzir a taxa de cruzamentos e, conseqüentemente, diminuir a disseminação da resistência. A resistência ao glyphosate em Lolium rigidum é transmitida via pólen e codificada por um gene nuclear (Powles et al., 2000; Lorraine-Colwill et al., 2001).

O maior número de sementes produzidas garante ao biótipo sensivel maior número de descendentes e a tendência de dominar o ambiente, se a pressão de seleção for retirada ou reduzida. Esta pressão é retirada quando não se utilizam, no local, herbicidas com o mecanismo de ação para o qual os biótipos adquiriram resistência - neste caso, o glyphosate. Assim, o manejo das plantas daninhas deve ser realizado com uso de outras práticas, como rotação de culturas e de métodos de controle, bem como de herbicidas com mecanismos de ação diferentes daquele para o qual as plantas possuem resistência (Mortimer, 1998).
Os resultados obtidos evidenciam Fator de Resistência de 16,8 e que o mecanismo que proporciona resistência ao glyphosate, no azevém, afeta a resposta dos biótipos resistentes aos graminicidas e interfere negativamente no acúmulo de matéria seca e na produção e seus componentes.

\section{LITERATURA CITADA}

BURNSIDE, O. C. Rationale for developing herbicideresistant crops. Weed Technol., v. 6, n. 3, p. 621-625, 1992.

HARKER, K. N.; DEKKER, J. Effects of phenology on translocation patterns of several herbicides in quackgrass (Agropyron repens). Weed Sci., v. 36, p. 463-472, 1988.

HARTZLER, B. Search for the glyphosate resistance mechanism in ryegrass. Disponível em: $<\mathrm{http}: / \mathrm{www}$. weeds/iastate.edu/mgmt $/ 2001 /$ serchformech.htm $>$. Acesso em: 26 mar. 2003.

LORRAINE-COLWILL, D. F. et al. Inheritance of evolved glyphosate resistance in Lolium rigidum (Gaud.). Theor. Appl. Genet., v. 102, p. 545-550, 2001.

LORRAINE-COLWILL, D. F. et al. Investigations into the mechanism of glyphosate resistance in Lolium rigidum. Pest. Biochem. Physiol., v. 74, p. 62-72, 2003.

MORTIMER, A. M. Review of graminicide resistance. Disponível em: <http://ipmwww.ncsu.edu/orgs/hrac/ monograph1.htm>. 1998. 32 p. Acesso em: 20 maio 1998.

PEREZ, A.; KOGAN, M. Glyphosate-resistant Lolium multiflorum in Chilean orchards. Weed Res., v. 43, p. 12-19, 2002.

POWLES, S. B. et al. Evolved resistence to glyphosate in rigid ryegrass (Lolium rigidum) in Australia. Weed Sci., v. 46 , p. $604-607,1998$.

POWLES, S. B. et al. Genetic and fitness of glyphosate resistant ryegrass. Crop updates. 2000. Disponível em: $<$ http://www. agric.wa.gov.au/cropupdates/2000/weeds/ Powles.htm>. Acesso em: 22 maio 2003.

PRATLEY, J. E. et al. Resistance to glyphosate in annual ryegrass (Lolium rigidum): I Bioevaluation. Weed Sci., v. 47, p. 405-411, 1999.

ROMAN, E. S. et al. Resistência de azevém (Lolium multiflorum) ao herbicida glyphosate. Planta Daninha, v. 22, n. 2, p. 301-306, 2004.

WEED Science. Official WSSA definitions. Disponível em: <http://www.weedscience.com /paper/definitions.htm>, 1 p. Acesso em: 22 abr. 1999.

WEED Science. Glycine (g/9) resistant weeds by species and country. Disponível em: $<$ http://www.weedscience.org/ Summary/UspeciesMOA.asp?1stMOAID = 12\&FmHRACGroup=Go>. Acesso em: 21 set. 2004. 